\title{
How can Einstein's curved space apply to earthquakes?
}

Junjin Huang ${ }^{1}$, Yuhan Lin ${ }^{1}$, Lipeng Liao', Chanyuk Lam ${ }^{1}$, Qiuyun Liu ${ }^{1}$,*

${ }^{1}$ School of Life Sciences, Sun Yat-Sen University, Guangzhou 510275, China.

*Correspondence author. School of Life Sciences, Sun Yat-Sen University, Guangzhou 510275, China.

E-mail address: liuqiuyunsysu@163.com 


\begin{abstract}
Einstein believed that space is curved, but the influence of solar and lunar gravitational pulls on seismic activities is different at different times of the day or of the month. Accelerations and decelerations of a large or a small mass on the Earth can appear at different times.
\end{abstract}


Seismic activity resembles weightlifting and is driven by solar and lunar gravitational pulls (1-3). In Einstein's view, space is curved. How to apply this to earthquakes remains an issue. At 0:00-12:00 am local time, solar pull accelerates earth-crust in the direction of the Earth's self-rotation, but at 12:00-0:00 pm local time, solar pull decelerates earth-crust opposite to the direction of the Earth's self-rotation. (1-3) This principle may account for different earthquake frequencies in different time of the day or of the month (4). The slower deceleration of a large mass or faster acceleration of a small mass creates strain on the earth-crust, similar to fast-traveling trains and cars on the same road with start or stops resulting in crashes (1-3).

\section{References}

1. Yan $\mathrm{S}$, et al. Earthquakes-an intricate trio dance of gravitation. Science e-letter. 2018; https://www.science.org/doi/10.1126/science.aag0482.

2. Yan S, et al. Volcanoes or earthquakes: Wrist wrestling. Science e-letter. 2019; https://www.science.org/doi/10.1126/science.358.6370.1520.

3. Liu, Q, et al. What Determines Focal Depth? OSF Preprints. 2021. https://osf.io/qgz85.

4. Nabhan David. The Journal of San Diego History. A Pattern of Seismicity in Southern California: The Possibility of Earthquakes Triggered by Lunar and Solar Gravitational Tides. 2013;59(3):157 